\title{
Parto e perinatalidade: $O$ papel do psicólogo hospitalar nesse contexto
}

\author{
Childbirth and perinatality: The role of the hospital psychologist in this context \\ Parto y perinatalidad: el papel del psicólogo hospitalario en este contexto
}

Recebido: 2200/04/2021 | Revisado: 01/04/2021 | Aceito: 04/05/2021 | Publicado: 29/05/2021

\author{
Thalyta Freitas dos Santos Laguna \\ ORCID: http://orcid.org/0000-0002-4227-0020 \\ Universidade Franciscana, Brasil \\ E-mail: thalytalaguna@gmail.com \\ Aline Priscila Subutzki Lemos \\ ORCID: https://orcid.org/0000-0002-0472-4474 \\ Universidade Franciscana, Brasil \\ E-mail: asubutzki@hotmail.com \\ Luísa Ferreira \\ ORCID: https://orcid.org/0000-0003-3285-5612 \\ Universidade Franciscana, Brasil \\ E-mail: luisa.lferreira@ufn.edu.br \\ Camila dos Santos Gonçalves \\ ORCID: http://orcid.org/0000-0002-0655-8895 \\ Universidade Franciscana, Brasil \\ E-mail: camilag@ufn.com.br
}

\begin{abstract}
Resumo
Atendimentos psicológicos realizados no contexto dos hospitais, clínicas e centros de saúde devem abranger os três pilares existentes no âmbito hospitalar, quais sejam, pacientes, familiares e profissionais da saúde. O psicólogo especialista na modalidade hospitalar pode atuar em diversas frentes, incluindo o atendimento individual e em grupos psicoterapêuticos, atendimentos em ambulatórios e unidades de terapia intensiva (UTIs), enfermarias, prontoatendimentos, avaliações diagnósticas, psicodiagnósticos e consultorias. Muito embora identifiquem-se diversos estudos que dissertam sobre a psicologia hospitalar, sobretudo no que tange à atuação junto ao doente, pouco se fala sobre essa frente no contexto do parto e da perinatalidade. Dito isto, este artigo tem por objetivo dissertar sobre o papel do psicólogo nesse contexto. Justifica-se por ser um estudo relevante, com pouca literatura sobre o tema, que busca trabalhar a importância do psicólogo não somente no tratamento de doentes, mas também na promoção de saúde das parturientes. Trata-se de um estudo qualitativo, com levantamento bibliográfico feito em bases de dados online. Os achados sugerem que apesar do psicólogo estar inserido no contexto hospitalar, sua inserção nos centros obstétricos ainda não é plenamente efetiva. Conclui-se, desta forma, que ainda há um longo caminho na construção de práticas psicológicas de apoio às famílias e, em especial às parturientes, antes, durante e após o parto, bem como, um espaço dentro do ambiente obstétrico/hospitalar para o profissional da psicologia. Outrossim, salienta-se a importância de pesquisas e estudos dedicados ao tema, que corroborem para a construção de protocolos relacionados ao atendimento psicológico no contexto obstétrico hospitalar.
\end{abstract}

Palavras-chave: Hospitalar; Obstétrica; Parto; Perinatal; Protocolos.

\begin{abstract}
Psychological assistance carried out in the context of hospitals, clinics and health centers must cover the three pillars existing in the hospital environment, namely, patients, family members and health professionals. The specialist psychologist in the hospital modality can act on several fronts, including individual care and in psychotherapeutic groups, outpatient care and intensive care units (ICUs), wards, emergency care, diagnostic assessments, psychodiagnostics and consultancies. Even though several studies that talk about hospital psychology are identified, especially with regard to working with the patient, little is said about this front in the context of childbirth and perinatality. That said, this article aims to talk about the role of the psychologist in this context. It is justified because it is a relevant study, with little literature on the subject, which seeks to work on the importance of the psychologist not only in the treatment of patients, but also in the health promotion of parturient women. It is a qualitative study, with bibliographic survey done in online databases. The findings suggest that although the psychologist is inserted in the hospital context, his insertion in obstetric centers is not yet fully effective. It is concluded, therefore, that there is still a long way to build psychological practices to support families and, in particular, parturients, before, during and after childbirth, as well as a space within the obstetric / hospital environment for the psychology professional.
\end{abstract}


Furthermore, it is emphasized the importance of research and studies dedicated to the theme, which corroborate for the construction of protocols related to psychological care in the hospital obstetric context.

Keywords: Hospital; Obstetric; Childbirth; Perinatal; Protocols.

\begin{abstract}
Resumen
La asistencia psicológica realizada en el contexto de hospitales, clínicas y centros de salud debe cubrir los tres pilares existentes en el ámbito hospitalario, a saber, pacientes, familiares y profesionales de la salud. El psicólogo especialista en la modalidad hospitalaria puede actuar en varios frentes, incluyendo la atención individual y en grupos psicoterapéuticos, atención ambulatoria y unidades de cuidados intensivos (UCI), salas, urgencias, valoraciones diagnósticas, psicodiagnósticos y consultas. Si bien se identifican varios estudios que hablan de psicología hospitalaria, especialmente en lo que respecta al trabajo con el paciente, poco se habla de este frente en el contexto del parto y la perinatalidad. Dicho esto, este artículo tiene como objetivo hablar sobre el papel del psicólogo en este contexto. Se justifica porque es un estudio relevante, con poca literatura sobre el tema, que busca trabajar sobre la importancia del psicólogo no solo en el tratamiento de las pacientes, sino también en la promoción de la salud de la parturienta. Es un estudio cualitativo, con levantamiento bibliográfico realizado en bases de datos en línea. Los hallazgos sugieren que si bien el psicólogo está insertado en el contexto hospitalario, su inserción en centros obstétricos aún no es del todo efectiva. Se concluye, por tanto, que aún queda un largo camino por construir prácticas psicológicas de apoyo a las familias y, en particular, a las parturientas, antes, durante y después del parto, así como un espacio dentro del ámbito obstétrico / hospitalario para el profesional de la psicología. Además, se enfatiza la importancia de las investigaciones y estudios dedicados al tema, que corroboran para la construcción de protocolos relacionados con la atención psicológica en el contexto obstétrico hospitalario.
\end{abstract}

Palabras clave: Hospital; Obstétrico; Parto; Perinatal; Protocolos.

\title{
1. Introdução
}

Segundo a Organização Mundial da Saúde (OMS), ter saúde significa gozar de bem estar físico, mental e social e vai muito além da ausência de doenças e enfermidades, constituindo um dos direitos fundamentais do cidadão (Brasil, 2020). Para além de um direito do indivíduo, a saúde compreende um dever do estado, que deve garanti-la integralmente através de políticas sociais (Conselho Regional de Psicologia [CRP], 2016). Nesse contexto, a atuação do psicólogo justifica-se em todas as frentes de saúde, e consubstancialmente, no contexto hospitalar.

Outrossim, a literatura sobre o tema aponta que os movimentos desses profissionais neste âmbito datam do início do século XIX, período no qual a psicologia hospitalar se fazia presente em unidades psiquiátricas. Da mesma forma, ressalta-se que desde a década de 1940 a política de saúde brasileira centra-se no hospital, porém somente em meados de 1990, passa-se a ter um crescente reconhecimento acerca da importância do psicólogo no contexto multiprofissional e interprofissional (Santos \& Vieira, 2012).

Queiroz, Azevedo, Cherer e Chatelard (2020) apontam que atendimentos psicológicos realizados no contexto dos hospitais, clínicas e centros de saúde devem abranger os três pilares existentes no âmbito hospitalar, quais sejam, pacientes, familiares e profissionais da saúde. Castro e Bornholdt (2004), baseados no Conselho Federal de Psicologia (2013), afirmam que o psicólogo especialista na modalidade hospitalar pode atuar em diversas frentes, incluindo o atendimento individual e em grupos psicoterapêuticos, atendimentos em ambulatórios e unidades de terapia intensiva (UTIs), enfermarias, pronto atendimentos, avaliações diagnósticas, psicodiagnósticos e consultorias. Santos e Vieira (2012) complementam que as atividades podem ser desenvolvidas em centros de oncologia, cardiologia, centros de terapia intensiva (CTIs), atendimentos pré-cirúrgicos, pós-cirúrgicos, acompanhamento de parturientes e acolhimento de recém-nascidos.

Muito embora identifiquem-se diversos estudos que dissertam sobre a psicologia hospitalar, sobretudo no que tange à atuação junto ao doente, pouco se fala sobre essa frente no contexto do parto e da perinatalidade. Arrais, Silva e Lordello (2014) afirmam que em artigos e livros, prepondera-se a importância do psicólogo no acompanhamento da gestação, pré-natal, pós parto, aleitamento e muitas vezes, em casos que evoluem para depressão pós parto ou que necessitaram do uso da UTI neonatal. Entretanto, evidenciam a escassez de estudos voltados a atuação da psicologia durante os trabalhos de parto, no parto, 
em possíveis intercorrências e em casos de óbitos perinatais. Arrais e Mourão (2013), baseadas em uma experiência de estágio acadêmico de pós-graduação em psicologia hospitalar, apontam em seu estudo que são poucos os casos para os quais os profissionais da psicologia são solicitados, detendo-se muitas vezes à casos de má formação em bebês, deficiências de qualquer ordem, intercorrências decorrentes do parto, em abortos e óbitos neonatais, sendo estes últimos causadores de maior sofrimento nas parturientes e na equipe.

A experiência do parto assume na vida da parturiente um potencial transformador, que se vivenciado em sua plenitude, pode inaugurar diversos marcos: entre ser filho(a) e tornar-se mãe, entre o bebê idealizado e o real, entre as expectativas geradas e a realidade vivida Vendrúscolo e Kruel (2016). Essa experiência passou por transformações importantes no decorrer do tempo; a gravidez, o parto e o nascimento que antes eram eventos que aconteciam no seio familiar com o suporte de pessoas que estavam ligadas à família, passou a ser um acontecimento hospitalar, conduzido através de suporte tecnológico, cirúrgico e com auxílio de médicos, enfermeiros e medicamentos de suporte (Souza, Gaíva \& Modes, 2011; Vieira et al., 2020). Isso posto, devido às crescentes transformações, a parturiente foi transformando-se em um sujeito que tem pouca autonomia e poder de decisão no que tange à maneira como o seu parto será conduzido e em virtude disso, o Ministério da Saúde preconiza o movimento de humanização do parto que visa, sobretudo, a prestação do serviço de assistência humanizada à parturiente, composto por uma série de ferramentas que visam o seu empoderamento antes, durante e depois do parto (Vendrúscolo \& Kruel, 2016).

Assim sendo, o profissional da psicologia pode exercer importante papel nesse contexto e desta forma, Arrais, Silva e Lordello (2014) apontam a relevância do psicólogo no contexto da perinatalidade, em diversas frentes, como: Acompanhamento psicológico durante o trabalho de parto e parto para alívio não farmacológico da dor, atendimento psicológico em casos de prematuridade e malformação fetal, atendimento psicológico em casos de óbito perinatal, atendimento psicológico a gestantes com hiperemese gravídica, atendimento psicológico à parturiente com diabetes, atendimento psicológico a parturiente com síndrome hipertensiva e atendimento psicológico aos acompanhantes das parturientes.

Destarte, o objetivo do presente estudo é dissertar sobre o papel do psicólogo hospitalar no contexto do parto e da perinatalidade, justificando-se sua relevância, uma vez que busca trabalhar a importância de psicólogos capacitados não somente no tratamento de doentes, mas também na promoção de saúde das parturientes.

\section{Metodologia}

Trata-se de uma pesquisa qualitativa, que é um método que procura entender e interpretar fenômenos sociais inseridos em determinado contexto (Bortoni-Ricardo, 2008). Outrossim, este tipo de pesquisa busca compreender um grupo ou fenômeno social, dando ênfase aos aspectos subjetivos que não podem ser quantificados (Augusto et al., 2013).

O presente estudo é um levantamento bibliográfico feito em bases de dados online, no qual todo material levantado foi escolhido e ordenado por meio de critérios previamente estabelecidos durante a definição dos objetivos do artigo. Acerca disso, Fantinato (2015) discute que este tipo de pesquisa se baseia em estudos realizados em fontes de dados documentais.

Isso posto, este estudo baseou-se na pergunta norteadora "Qual o papel do psicólogo hospitalar no contexto do parto e da perinatalidade?". Os materiais foram pesquisados com base na revisão narrativa da literatura, que tem como objetivo descrever e discutir o desenvolvimento do estado da arte partindo de pressupostos teóricos (Bardin, 2016).

As palavras chaves utilizadas foram: Hospitalar; Obstétrica; Parto; Perinatal; Protocolos. A coleta de dados se deu por meio de livros, portais como o do Conselho Regional de Psicologia e do Ministério da saúde, bem como, de artigos científicos extraídos de revistas nacionais e internacionais nas plataformas Scientific Eletronic Library Online (Scielo) e Literatura Latino Americana e do Caribe em Ciências da Saúde (Lilacs). 
Destarte, realizou-se uma análise de conteúdo que apresenta a seguinte ordem: organização, codificação, categorização, tratamento e interpretação dos resultados (Bardin, 2016). Os achados encontrados na literatura foram discutidos em três categorias respectivas: (1) O contexto do parto: do domicílio ao hospital; (2) A humanização do nascimento; e (3) O papel do psicólogo no contexto do parto e da perinatalidade.

\section{Resultados e Discussão}

\section{O contexto do parto: do domicílio ao hospital}

Desde os primórdios, para a mulher, o parto é um evento marcante, carregado de sentimentos e de identidade; ele é e sempre foi único e pertencente à pessoa que gesta. Entretanto, a hospitalização do parto trouxe consigo muitos fatores novos, incluindo a presença do homem. Frente a isso, Del Priore (1995) afirma que:

Esta coisa de mulher, em que se constituía dar à luz, requeria ritos e saberes próprios, em que os homens só interfeririam em casos de emergência e, sobretudo, nos centros urbanos. A presença masculina no parto era desconfortável, nem sempre bem-vinda, porque, além dos médicos mostrarem-se em seus relatos absolutamente insensíveis à dor das parturientes, as mulheres pareciam também atingidas pelo tabu de mostrar seus genitais, preferindo, por razões psicológicas e humanitárias, a companhia das parteiras (Del Priore, 1995, p. 263).

Além da presença de médicos, enfermeiros e das equipes de maneira geral, na nova era do parto houve a inserção da medicalização, bem como de formas não naturais de parir. O fórceps foi inserido ao nascimento como forma de salvar a vida dos bebês e das mães em uma época em que a cesárea ainda era sinônimo de risco de vida para ambos. Um século depois, a cesariana já não representava mais tanto perigo de óbito materno e passou a ser incluída e enfatizada nos nascimentos ocorridos no Brasil (Maldonado, 2002).

A cesárea, ainda segundo a mesma autora, é o nome dado ao ato cirúrgico hospitalar que se constitui através da incisão do abdômen e da parede do útero da gestante para a retirada do bebê, prática que fez com que o fórceps fosse deixado em segundo plano. Sobre o parto vaginal, ou também chamado de parto normal, abarca diversas posições: o parto preparado, nasceu em berço russo com o objetivo de treinar a parturiente para participar ativamente desse momento de forma lúcida e cooperativa, sem nenhuma ou pouca analgesia. Já o parto vertical utiliza as posições verticais, sentada, de pé, de joelhos ou de cócoras; é considerado um dos mais antigos modos de parir e ocorre desde o antigo Egito e na Grécia antiga, entre os romanos (Maldonado, 2002).

A entrada da mulher no hospital pôs fim a feminização do parto; o autor afirma que a inserção dos médicos e seus instrumentos não naturais levou as parteiras para fora de cena e deslegitimou as parturientes dos eventos que o parto envolvia Osava (1997). É importante ressaltar ainda que um dos principais fatores que levaram a rápida legitimação dos médicos, foi o terror do período de caça às bruxas que ocorreu entre os séculos XIV e XVII, momento no qual as parteiras passaram a incomodar as autoridades em busca de uma assistência intervencionista, uma vez que amenizava as dores das mulheres na hora do parto em uma tenebrosa época em que se acreditava que as mulheres deveriam sofrer em decorrência do pecado original. (Spink, 2013).

A mulher, que agora dava à luz no hospital em meio às novas tecnologias, não mais é percebida como sujeito, mas sim como objeto do processo do modelo biomédico. Na Conferência Nacional sobre a Organização de Serviços para Maternidade Segura à Luz da Humanização realizada em 1998, Lima (1998) apresenta sua visão sobre o paradigma entre o modelo biomédico e o modelo holístico:

O modelo biomédico vê o nascimento como um processo patológico, é um modelo altamente intervencionista, que tem no hospital o seu centro de atenção e ênfase no uso invasivo de tecnologia sofisticada. E o modelo holístico que 
vê o nascimento como um processo natural, um processo que além de envolver os fenômenos biológicos, envolve os fenômenos psíquicos, econômicos, sociais, culturais e que não pode ser tratado apenas como um evento médico. Ele tem na comunidade, na família, no domicílio, o centro da sua atenção e não no hospital. (Lima, 1998, p. 71).

Frente a todas as questões que circundam as parturientes nesse novo contexto, fez-se necessária a implementação da humanização do parto. Isso posto, dentre tantos outros recursos, a presença de um acompanhante de escolha da mulher se torna imprescindível, com o objetivo principal de dar suporte durante o parto. Essa prática é recomendada e preconizada pela Organização Mundial de Saúde e tem sido desenvolvida com êxito em diversos países (Domingues, 2002).

\section{A humanização do nascimento}

Segundo Deslandes (2004), há décadas, as iniciativas que se identificam com a humanização do parto e com os direitos das mulheres vêm se tornando pauta dos movimentos feministas em saúde. Contudo, a humanização da assistência faz parte de um propósito mais direcionado ao atendimento de bebês de baixo peso, internados em UTI. Projetos como "Maternidade Segura" e "Método Canguru", são amplamente apoiados pelo Ministério da Saúde e pela Organização Mundial de Saúde, considerados referência para a rede pública.

Humanizar é um termo que nos remete à assistência valorizadora da qualidade do cuidado, associada ao reconhecimento dos direitos do paciente, sua subjetividade e referências culturais. Outrossim, inclui também a valorização do profissional presente e do diálogo existente intra e interequipes, envolvendo todo contato presente dentro do hospital, seja no atendimento e cuidado com o paciente, seja no olhar para a equipe e para cada profissional ali atuante (Deslandes, 2004).

Há muitos anos os hospitais perderam o caráter acolhedor que o dispositivo religioso lhe conferia anteriormente. Goffman (1961) como citado em Crepaldi (1999), já chamava de "processo de despojamento", no qual a pessoa, no momento que entra no hospital, é transformada em uma doença, um número de leito. Até hoje presenciamos a desumanidade do profissional da saúde no ambiente de trabalho. É preciso desenvolver uma reflexividade crítica para que essas práticas venham a produzir efeitos transformadores e aperfeiçoem o a qualidade dos serviços; é necessário acabar com a "prática do silêncio", que compreende o indivíduo isolado da sociedade. Com o surgimento das revisões e atualizações, o novo modelo profissional - a partir do que está sendo evidenciado - tanto ao nível de formação profissional quanto de estratégias de inserção dos psicólogos, deve elaborar um indivíduo que considere a ação histórica dos homens (Carvalho, 2013; Goffman, 1961 como citado em Crepaldi, 1999; Moura, 2003 como citado em Castro e Bornholdt, 2004.).

A definição de atenção humanizada é vasta e envolve um conjunto de conhecimentos, práticas e atitudes que se destinam a promoção do parto e do nascimento saudáveis, prevenindo a morbimortalidade materna e perinatal. Este cuidado inicia-se no pré-natal prolongando-se até o puerpério, e tem como objetivo garantir que a equipe de saúde irá realizar apenas procedimentos comprovadamente benéficos para a mulher e o bebê, evitando intervenções desnecessárias e preservando sua privacidade e autonomia (Brasil, 2001).

A mãe e a família devem ser o "centro" do processo de nascimento. A equipe médica deve proporcionar um ambiente acolhedor de carinho e atenção, onde a mulher vai receber o apoio e ter suas dúvidas e anseios respondidos de forma clara e acessível. O ambiente da maternidade não pode ser hostil, com rotinas pesadas e normas inflexíveis que envolvam apenas os interesses do serviço e da equipe de saúde, e também não deve ser um local onde a mulher não sinta liberdade para manifestar seus sentimentos, angústias e medos. Destarte, a mulher precisa se sentir segura, amparada e ter presente o pai do bebê ou uma pessoa da família, uma amiga, permitindo que o momento se torne uma experiência de alegria e amor (Gaíva \& Tavares, 2002).

O processo do nascimento é singular, uma vivência única na vida da mãe e de seu parceiro, e que envolve além deles, suas famílias e a comunidade. A gravidez e o parto são eventos sociais que envolvem uma experiência humana significativa e 
enriquecedora. Os profissionais envolvidos são apenas coadjuvantes e desenvolvem um papel muito importante, com o compromisso de colocar seu aprendizado a serviço do bem-estar da mulher e do seu bebê, reconhecendo os momentos em que são necessárias intervenções para assegurar a saúde da mãe do recém-nascido. Estes profissionais têm uma grande responsabilidade em suas mãos e realizam um trabalho admirável, podendo minimizar a dor, ficar ao lado, dar conforto, esclarecer, orientar e auxiliar na hora do parto e nascimento. São eles os primeiros a tocarem em cada ser que nasce e devem ter consciência de sua função e pertença (Brasil, 2001).

Além disso, segundo Klaus e Kennel (1993), faz parte do parto humanizado o contato pele-pele, sendo um dos mais fortes canais de estabelecimento da relação mãe-filho. Eles comentam sobre a existência de um momento vulnerável, logo após o nascimento, onde a separação poderia trazer problemas futuros na formação de vínculos afetivos entre mãe e filho. O toque precoce entre estes na sala de parto é necessário para a formação do apego, uma vez que o recém-nascido, no pós-parto, encontra-se em um período entendido como inatividade alerta - os primeiros 30 a 60 minutos após o nascimento - quando o bebê responde aos estímulos externos como ver, ouvir e mover-se de acordo com a voz materna.

Para Souza, Gaíva e Modes (2011), é neste momento também que se apresentam habilidades sensoriais e motoras que são capazes de estimular um meio de comunicação com os pais, que são extremamente importantes para a criação do vínculo. Por isso a importância de o recém-nascido estar em contato com a mãe logo após o seu nascimento, para que o vínculo seja estabelecido (Souza, Gaíva \& Modes, 2011).

Ainda segundo os autores, a humanização na assistência ao nascimento não é uma experiência presente na maioria dos hospitais. Grande parte dos profissionais não estão preparados para prestar um atendimento humanizado e com qualidade, tanto para a mãe quanto para o recém-nascido e, desta forma, fica explícita a necessidade de investimentos na capacitação dos profissionais que atuam no processo de nascimento, enfocando não apenas em tecnologias adequadas ao atendimento ao parto e ao recém-nascido, mas também às questões atuais da humanização no processo de nascimento (Souza, Gaíva, \& Modes, 2011).

Nos últimos anos, avanços tecnológicos e terapêuticos têm contribuído para a diminuição do número de mortes perinatais. Todavia, são estes mesmos avanços que acabaram por desconsiderar os valores humanos e consequentemente inserir, na conduta do atendimento ao paciente, um padrão técnico, que desumanizou a assistência. À vista disso, o atendimento preconizado ao bebê pelo hospital desvaloriza os aspectos emocionais provenientes da experiência do momento do nascimento (Gaíva \& Tavares, 2002).

As maternidades tornaram-se instituições com grande poder de decisão e imposição sobre as mães e suas famílias. São elas que definem como e quando será o parto, qual o comportamento esperado dos envolvidos, quando e com quem pode ser realizado o contato com o binômio mãe-filho. Isso demonstra uma falta de consideração por parte dos profissionais da área da saúde para com a experiência das famílias, uma vez que os profissionais utilizam instrumentos, materiais e técnicas existentes sem levar em conta o saber, a prática, assim como o contexto cultural onde as aquelas estavam inseridas, tornando-se o parto um momento invasivo e desconhecido para a mulher (Gaíva \& Tavares, 2002; Monticelli, 1994).

Isso posto, é preciso pensar no conhecimento e articulação de todos os fatores envolvidos no processo saúde-doença (Almeida, 2000). Consubstancialmente, evidencia-se a importância do psicólogo no ambiente hospitalar, visto que este profissional, ao integrar a equipe de saúde, deve favorecer o funcionamento interdisciplinar, facilitando a comunicação entre seus membros, quando necessário. O seu trabalho com as pacientes na maternidade não se relaciona apenas no sentido de resolução de conflitos, mas também na promoção da saúde.

\section{O papel do psicólogo hospitalar no contexto do parto e da perinatalidade}

O termo psicologia perinatal foi adotado para substituir os termos que surgiam no contexto hospitalar. A exemplo 
disso, tem-se a psicologia obstétrica, que indicava algo mais ligado à humanização dos ambientes obstétricos do que propriamente a área médica. A partir dessa diferenciação, entende-se que a psicologia perinatal trata de ampliar o acolhimento para além do momento do parto, abrangendo todo o ciclo perinatal de pré e pós-natal, além do parto em si, desenvolvendo um trabalho específico nos centros obstétricos (Iaconelli, 2012).

Para Arrais, Silva e Lordello (2014), um dos desafios é construir uma demanda em torno da perinatalidade, visto que a chegada de um bebê e o ciclo gravídico são vistos sempre com olhar positivo e são eventos que só trazem felicidade, o que acaba ofuscando o sofrimento pré-natal e todas as demandas que podem surgir no pós-natal como o baby blue, problemas com amamentação e possíveis causalidades como complicações fisiológicas da puérpera, certas deformações do recém-nascido e até mesmo o óbito.

No ambiente de obstetrícia, a psicologia é amplamente acionada em casos em que as equipes responsáveis são postas em situações adversas e que acabam gerando muita comoção ou sentimento de impotência. Esse tipo de conduta acaba gerando uma estigmatização e certo preconceito em relação à atuação do psicólogo em ambientes hospitalares de obstetrícia (Iaconelli, 2012). A partir desse pensamento de que a psicologia é uma ciência voltada apenas ao luto e às demandas de desconforto da equipe obstétrica, a psicologia se reinventa como promotora de saúde mental no ambiente de obstetrícia. Nesse sentido, preocupa-se com o acolhimento psicológico da gestante, atuando desde o acompanhamento dos níveis de stress e de ansiedade, até a educação da gestante, da parturiente e da puérpera para as adversidades do ciclo perinatal (Consonni, 2019).

Arrais, Silva e Lordello (2014) relatam, após a experiência de quatro anos de atuação no centro obstétrico de uma grande maternidade pública de Brasília, a importância do psicólogo no contexto da perinatalidade. O psicólogo hospitalar em um centro obstétrico deve trabalhar com os aspectos biopsicossociais, realizando atendimentos psicoterápicos diversos, discussão de casos, orientações terapêuticas, supervisão de parto, manejo da dor, protocolo de luto e encaminhamentos, sempre atentando para saúde mental e singularidade dos pacientes e familiares.

Outrossim, a partir da prática construída ao longo dos quatro anos de experiência na área da obstetrícia vivenciada pelos autores acima citados, percebeu-se que o profissional da psicologia pode atuar em diversas frentes no âmbito hospitalar/obstétrico, quais sejam: acompanhamento psicológico durante o trabalho de parto e parto para alívio não farmacológico da dor, atendimento psicológico em casos de prematuridade e malformação fetal, atendimento psicológico em casos de óbito perinatal, atendimento psicológico a gestantes com hiperemese gravídica, atendimento psicológico à parturiente com diabetes, atendimento psicológico a parturiente com síndrome hipertensiva e atendimento psicológico aos acompanhantes das parturientes (Arrais, Silva \& Lordello 2014).

Isso posto, constatou-se que apesar do psicólogo estar inserido no contexto hospitalar, sua inserção efetiva nos centros obstétricos está em construção. Então vale ressaltar que o psicólogo, em sua competência, deve investir em comunicação empática e escuta ativa, para que através das suas intervenções, possa mostrar o sentido de sua atuação. Assim, progressivamente, poderá sensibilizar a equipe e fazê-la entender a contribuição da psicologia para a área da saúde, revertendo o quadro de desvalorização para o conhecimento e aceitação da atuação psicológica perinatal.

\section{Considerações Finais}

Esta pesquisa se propôs, como objetivo geral, dissertar sobre o papel do psicólogo hospitalar no contexto do parto e da perinatalidade. Nesse sentido, buscou ampliar os estudos acerca desse tema através da revisão de bibliografias que contemplam a história do parto e a humanização do nascimento, trazendo a clareza de como ao longo dos anos foi se perdendo a subjetivação da parturiente através dos modelos biomédicos e, então, denunciando a importância do psicólogo ser inserido no 
âmbito do parto e da perinatalidade, visto que o papel do psicólogo sempre será, preservar a saúde mental e a subjetividade dos pacientes.

Através da história do parto, pôde-se perceber que o acompanhante de convivência, escolha e confiança da mulher, foi retirado do cenário no transcorrer dos tempos e, aos poucos, está sendo resgatado. Ao longo da história, o parto passou de um evento que ocorria em casa para uma intervenção hospitalar, as parteiras que assistiam as mulheres foram substituídas por médicos, houve a inserção de instrumentos e da medicalização, e assim, passou de uma ocorrência natural a um evento regrado. Na humanização do parto, compreendemos que é indispensável que ocorram mudanças no modelo biomédico, essencialmente técnico, para um que valorize os aspectos sociais e culturais da gestação e parto, de maneira que mulheres/famílias tenham a experiência de um parto verdadeiramente humanizado.

Devido ao número reduzido de estudos com enfoque no papel do psicólogo hospitalar no contexto do parto e da perinatalidade, faz-se necessário desenvolver novos trabalhos para maior esclarecimento das rotinas, possibilidades de abordagem e atribuições para os psicólogos(as) que desejam atuar nesse campo.

\section{Referências}

Almeida, E. C. (2000). O Psicólogo no Hospital Geral. Psicologia: Ciência e Profissão, 20, $24-27 . \quad$ Recuperado de: http://pepsic.bvsalud.org/pdf/pcp/v20n3/v20n3a05.pdf

Arrais, A. da R. \& Mourão, M. A. (2013). Proposta de atuação do psicólogo hospitalar em maternidade e UTI neonatal baseada em uma experiência de estágio. Revista Psicologia e Saúde, 152-164. DOI: https://doi.org/10.20435/pssa.v5i2.284

Arrais, R., Silva. N. O. Lordello, S. R. M. (2014). Perception Obstetrical Team on the Role of Psychologist in a Hospital Center of Obstetric. 49-67. DOI: https://doi.org/10.33362/ries.v3i2.288

Augusto, C. A. et al. (2013). Pesquisa Qualitativa: rigor metodológico no tratamento da teoria dos custos de transação em artigos apresentados nos congressos da Sober Revista de Economia e Sociologia Rural, v.51, n.4, p. 745-764, 2013. DOI: https://dx.doi.org/10.1590/S0103-20032013000400007

Bardin, L. (2016). Análise de conteúdo. São Paulo: Edições 70.

Bortoni-Ricardo, S. M. (2008) O professor pesquisador: introdução à pesquisa qualitativa. São Paulo: Parábola Editorial.

Castro, E. K. de, \& Bornholdt, E. (2004). Psicologia da saúde x psicologia hospitalar: definições e possibilidades de inserção profissional. Psicologia: Ciência e Profissão, 24(3), 48-57. DOI: https://doi.org/10.1590/s1414-98932004000300007

Carvalho, D. B. (2013). Psicologia da Saúde Crítica no Contexto Hospitalar. Psicologia: Ciência e Profissão, 33(2), 350-365. DOI: https://doi.org/10.1590/S1414-98932013000200008

Conselho Regional de Psicologia - PR (2016). Caderno de psicologia hospitalar: considerações sobre assistência, ensino, pesquisa e gestão. Recuperado de: https://crppr.org.br/wp-content/uploads/2019/05/AF_CRP_Caderno_Hospitalar_pdf.pdf

Consonni, E. B. (2019). A psicologia perinatal frente aos diversos desafios da maternidade. Salvador. Recuperado de: http://schenautomacao.com.br/psico/envio/files/685.pdf

Crepaldi, M. A. (1999). Bioética e Interdisciplinaridade: direitos de pacientes e acompanhantes na hospitalização. Ribeirão Preto: Paidéia.

Del Priore M. (1995). Ao sul do corpo: condição feminina, maternidades e mentalidades no Brasil colônia. Rio de Janeiro: José Olympio $2^{a}$.ed.

Deslandes, S. F. (2004). Análise do discurso oficial sobre a humanização da assistência hospitalar. Ciência \& Saúde Coletiva, 9 (1), 7-14. DOI: https://doi.org/10.1590/51413-81232004000100002

Domingues, R. M. S. M. (2002). Acompanhantes familiares na assistência ao parto normal: a experiência da maternidade Leila Diniz. Dissertação de Mestrado, Departamento de endemias, ambiente e sociedade, Fundação Osvaldo Cruz, Rio de Janeiro. Disponível em: https://www.arca.fiocruz.br/handle/icict/5214

Fantinato, M. (2015). Métodos de pesquisa. São Paulo: USP.

Gaíva, M. A. M., \&Tavares, C. M. A. (2002). O nascimento: um ato de violência ao recém-nascido? Porto Alegre: Rev. Gaúcho Enferm., 23(1), 132-145. Recuperado de: https://seer.ufrgs.br/RevistaGauchadeEnfermagem/article/view/4408

Iaconelli, V. (2012) O que é psicologia perinatal: definição de um campo de estudo e atuação. Área de Estudos do Instituto Brasileiro de Psicologia Perinatal. disponível em http://www.institutogerar.com.br/

Klaus, M. H., \& Kennel, J. H. (1993). Pais/Bebê: a formação do apego. Porto Alegre: Artes Médicas. 
Research, Society and Development, v. 10, n. 6, e21510615351, 2021

(CC BY 4.0) | ISSN 2525-3409 | DOI: http://dx.doi.org/10.33448/rsd-v10i6.15351

Lima J. B. (1998). Práticas obstétricas para maternidade segura: guia da OMS. In: MS/ SESA/ JICA. Relatório da Conferência Nacional sobre Organização de Serviços para Maternidade Segura à Luz da Humanização - Projeto Luz. Fortaleza: Ministério da Saúde/ Secretaria Estadual de Saúde / Missão Japonesa no Ceará, p.70-6.

Maldonado, M. (2002) . Psicologia da Gravidez: parto e puerpério. São Paulo: Saraiva, 16. ed.

Ministério da Saúde. (2001). Parto, aborto e puerpério. Brasília. 2a ed. Recuperado de: https://bvsms.saude.gov.br/bvs/publicacoes/cd04_13.pdf

Ministério da Saúde (2020). O que significa ter saúde? Portal Saúde Brasil. Recuperado de: https://saudebrasil.saude.gov.br/eu-quero-me-exercitar-mais/oque-significa-ter-saude

Monticelli, M. (1994). O nascimento como um rito de passagem: uma abordagem cultural para o cuidado de enfermagem às mulheres e recém-nascidos. (Dissertação de Mestrado). Florianópolis: UFSC. Recuperado de: http://repositorio.ufsc.br/xmlui/handle/123456789/76041

Osava R. H. (1997). Assistência ao parto no Brasil: o lugar do não médico (tese de doutorado). São Paulo: Faculdade de Saúde Pública/ USP. Recuperado de: https://www.teses.usp.br/teses/disponiveis/6/6136/tde-10032020-120733/en.php

Queiroz, L. L. G. de, Azevedo, A. P. B., Cherer, E. de Q., \& Chatelard, D. S. (2020). Psychology at hospital maternity: an experience report. Fractal: Revista de Psicologia, 32(1), 57-63. DOI: https://doi.org/10.22409/1984-0292/v32i1/5679

Santos, L. de J., \& Vieira, M. J. (2012). Atuação do psicólogo nos hospitais e nas maternidades do estado de Sergipe. Ciencia e Saude Coletiva, 17(5), 11911202. DOI: https://doi.org/10.1590/S1413-81232012000500013

Souza, T. G. de, Gaíva, M. A. M., \& Modes, P. S. S. dos A. (2011). A humanização do nascimento: percepção dos profissionais de saúde que atuam na atenção ao parto. Revista Gaúcha de Enfermagem, 32(3), 479-486. DOI: https://doi.org/10.1590/s1983-14472011000300007

Spink, M. J. (2013) Psicologia Social e Saúde: saberes e sentidos. Rio de Janeiro: Vozes, 9. ed.

Vendrúscolo, C. T., \& Kruel, C. S. (2016). A história do parto: do domicílio ao hospital; das parteiras ao médico; de sujeito a objeto. Disciplinarum Scientia | Ciências Humanas, 16(1), 95-107. Recuperado https://periodicos.ufn.edu.br/index.php/disciplinarumCH/article/view/1842/1731\%0Ahttp://www.periodicos.unifra.br/index.php/disciplinarumCH/article/view/ $1842 / 1731$

Vieira, M. J. de O., Santos, A. A. P. dos., Sanches, M. E. T. de L., Silva, J. M. de O., Oliveira, K. R. V. de. (2020). Representações sociais de mulheres frente à escolha da via de parto. Research, Society and Development, v. 9, n. 11, e3189119840. 